\title{
MEDICINA
}

\section{Interacción de factores ambientales y genéticos asociados con el desarrollo del cáncer de ovario}

\author{
Francisco Benítez-Capistros ${ }^{1,2}$, Ricardo Benítez-Capistros ${ }^{3}$, Carolina Echeverría ${ }^{4}$, Fabián Oña- \\ Cisneros ${ }^{4}$, César Paz-y-Miño ${ }^{4}$, Andrés López-Cortés ${ }^{4}$ \\ ${ }^{1}$ Laboratory of Plant Biology and Nature Management (APNA), Faculty of Sciences and Bio-engineering Sciences, Vrije \\ Universiteit Brussel (VUB), Brussels, Belgium \\ ${ }^{2}$ Laboratory of Systems Ecology and Resource Management, Faculty of Sciences, Department of Organism Biology, \\ Université Libre de Bruxelles (ULB), Brussels, Belgium \\ ${ }^{3}$ Empresa Petroamazonas EP. Departamento de Salud, Seguridad y Ambiente, Quito, Ecuador \\ ${ }^{4}$ Instituto de Investigaciones Biomédicas, Facultad de Ciencias de la Salud, Universidad de las Américas, Quito, Ecuador
}

andres.lopez@udla.edu.ecＲecibido: 2014-05-29; aceptado: 2014-07-02

\section{RESUMEN.-}

El cáncer de ovario es la neoplasia maligna ginecológica más letal debido a su asintomatología y etapa avanzada de diagnóstico. La tasa de incidencia a nivel mundial es de 6,3 por cada 100.000 mujeres, presentando las tasas más altas en zonas geográficas industrializadas como Europa $(10,1)$, América del Norte $(8,7)$ y Oceanía $(7,6)$. Entre los factores de riesgo asociados con el desarrollo de este cáncer se encuentran: edad avanzada, niveles descontrolados de carga hormonal, obesidad, antecedentes familiares de cáncer, exposición a agentes carcinogénicos, presencia de agentes patógenos y mutaciones genéticas. Con respecto al perfil genético, la presencia de mutaciones en los diferentes biomarcadores moleculares (supresores de tumores, oncogenes, genes de apoptosis y reparadores del $\mathrm{ADN}$ ) aumenta el riesgo de desarrollar cáncer de ovario. Entre los genes más representativos están: $B R C A 1, B R C A 2, T P 53, R A D 51$ y VDR. Además, el correcto tratamiento de esta enfermedad dependerá del estadio tumoral, edad, capacidad de absorción de fármacos, histopatología, carga hormonal y perfil genético. Entre los tratamientos más aplicados se encuentra la cirugía, los medicamentos homeopáticos; la aplicación de fármacos, la aplicación de micro ARNs y de inhibidores multicinasas. En conclusión, el desarrollo del cáncer (ovario) dependerá de la interacción de cuatro variables relevantes: (1) el tiempo y (2) la dosis de exposición a los carcinógenos, (3) la edad avanzada, y (4) la predisposición genética. Es decir, los factores ambientales asociados con los factores genéticos predisponen al desarrollo de cáncer.

PALABRAS CLAVES: ambiente, cáncer de ovario, factores de riesgo, genes, tratamiento

\section{ABSTRACT.-}

Ovarian cancer is the deadlist gynecological neoplasia due to its asymptomatology and its advanced stage of diagnosis. The incidence rate worldwide is at 6.3 for each 100,000 women, 
where the highest rates are found in the industrialized geographic areas, such as Europe (10.1), North America (8.7) and Oceania (7.6). Some of the risk factors that are mostly associated with the development of this type of cancer are: advanced age, uncontrolled levels of hormone loading, obesity, family history of cancer, exposure to carcinogenic agents, presence of pathogenic agents and genetic mutations. Regarding genetic profiling, the presence of mutations in different molecular biomarkers (tumor supressors, oncogenes, apoptotic genes and DNA repairs) increases the risk to develop ovarian cancer. Among the most representative genes are: $B R C A 1, B R C A 2, T P 53$, RAD51 and VDR. On the other hand, the correct treatment carried out for this disease will depend on the tumor stage age, capacity to absorb medicine, histopathology, hormonal loading and genetic profiling. The most common treatments are: surgery, homeopathic medicaments, drugs, micro RNA's and multikinase inhibitors application. In conclusion, the development of cancer (ovarian) will depend on four relevant variables: (1) time and (2) exposure dose to carcinogens, (3) advanced age, and (4) genetic predisposition. In other words, the association between the environmental factors and the genetic factors triggers the development of cancer.

KEYWORDS: environment, genes, ovarian cancer, risk factors, treatment

\section{INTRODUCCIÓN}

La humanidad ha tratado de dominar la "vida", metafóricamente, en una lucha para vencer a la muerte. Los seres humanos están sufriendo las consecuencias de sus propias acciones, las mismas que no solo reflejan la degradación ambiental que ha sufrido la Tierra, sino también la propia decadencia de la salud humana. La incidencia de muchas enfermedades relacionadas con el ambiente, como el cáncer, ha crecido y ha sido bien documentada en las últimas décadas (Clavel, 2007; Nicolopoulou-Stamati et al., 2004).

La proliferación y diferenciación celular constituyen proceso normal del organismo tanto en el proceso de embriogénesis como en la regeneración tisular. El ADN celular está conformado por genes supresores de tumores, proto-oncogenes, genes reparadores y genes de apoptosis, los cuales controlan el correcto crecimiento, división y muerte celular (Chen et al., 2011). Cuando el individuo se encuentra expuesto a diversos factores epigenéticos no solo existen mutaciones en la doble cadena de ADN sino también existen cambios en los patrones de expresión génica como por ejemplo: la alteración de la cromatina generada por la metilación de los residuos de citosina en los dinucleótidos CpG, la disfunción de proteínas histonas a través de procesos anormales de metilación y acetilación y la generación del silenciamiento epigenético mediante proce- sos de metilación en regiones encargadas de la transcripción génica de proteínas protectoras del ADN. Estos cambios en los patrones de expresión génica conducen a una disfunción fisiológica, displasia histológica, formación de tumores y desarrollo del cáncer (American Cancer Society, 2013; Wrzeszczynski et al., 2011; Lagos y Soto, 2007; Hernández, 1999).

El ovario anatómicamente está formado por el mesosálpinx que se proyecta desde el mesoovario hacia la parte del infundíbulo en las trompas uterinas, el mesoovario que es el mesenterio magno del útero y un pliegue del peritoneo, y el mesometrio que se encuentra inferior al mesosálpinx y al mesoovaario (Moore, 2010). Estas estructuras conforman el ligamento ancho, el cual es la porción de mesenterio que se dirige hacia los ovarios a través del mesoovario. Además, posee el ligamento propio, el cual los fija al útero y transcurre hacia la parte media del mesoovario, el ligamento suspensorio que los mantiene unidos a la cavidad pelviana y el hilio, donde entran y salen los nervios, vasos sanguíneos y linfáticos (Moore, 2010). El ovario, cuya longitud va de 2,5 a 4,5 cm y diámetro va de 0,5 a $1 \mathrm{~cm}$ (Karst y Drapkin, 2010), cumple con funciones de desarrollo de células germinales y producción de hormonas necesarias para la reproducción como el estrógeno y la progesterona (Hernández, 1999). 
El cáncer de ovario, también conocido como asesino silencioso, es la neoplasia maligna ginecológica más letal debido a que es asintomática y generalmente se la diagnostica en una etapa avanzada (Kwon y Kee-Shin, 2011; Bankhead et al., 2008; Chobanian y Dietrich, 2008). La ubicación tumoral tiene relación con el tipo de célula afectada, los tumores epiteliales se producen en las células que cubren la superficie del ovario, los germinales se producen en las células que originan los óvulos y los estromales se producen en las células que dan soporte y originan las hormonas femeninas (Chobanian y Dietrich, 2008; Hernández, 1999). Las células cancerígenas, al proliferarse sin control, pueden invadir los nódulos del hilio renal, el parénquima del hígado o los pulmones mediante la circulación sanguínea y linfática, proceso al cual se lo denomina metástasis (National Cancer Institute, 2013; Bast et al., 2009).

El pronóstico de esta enfermedad es muy pobre debido a que al ser asintomático, la mayoría de pacientes acuden al médico en un estadio avanzado del cáncer. A pesar de que la carga genética hereditaria solo representa el $15 \%$ de los pacientes, el diagnóstico temprano es útil si se lo realiza a mujeres que tengan antecedentes familiares de esta enfermedad (Sánchez, 2005). Además, es importante tomar en cuenta que el cáncer de ovario puede desencadenar una sintomatología similar a una enfermedad gastrointestinal como dolor y distensión abdominal, mayor frecuencia urinaria y pérdida de apetito (Patel, 2012).

\section{Histopatología.-}

Los tumores ováricos comprenden una variedad de características histopatológicas y se subdividen en: mucinoso, seroso, endometrioide, de células claras y de células de transición. Entre estos grupos de tumores, se distinguen tres categorías de acuerdo con el comportamiento biológico: benigno, limítrofe y maligno (Smolle et al., 2013; Kurman and Shih, 2008).

El carcinoma mucinoso primario se caracteriza por una gran masa ovárica unilateral con una superficie externa lisa, con un patrón de crecimiento expansivo, con un patrón papilar complejo y con restos necróticos e histológicos semejantes a tumores limítrofes (Lee et al., 2003). El carcinoma mucinoso metastásico es bilateral y muestra una superficie externa multinodular, la superficie de corte de la lesión metastásica varía de sólido a multiquística, imitando la masa de ovario primario (Lee et al., 2003).

En la categoría de tumor limítrofe, el tipo seroso y la variante endocervical del tipo mucinoso se puede presentar en las etapas avanzadas II y III y están asociados con la recurrencia en aproximadamente el 10\% de los casos (Smolle et al., 2013).

Si se consideran que los otros tipos histológicos se presentan en el estadio I y muestran una evolución benigna, las neoplasias serosas son la categoría más frecuente que abarca más del $50 \%$ de los tumores de ovario. Actualmente, los carcinomas serosos se distancian histológica y biológicamente en dos subtipos, los de bajo y alto grado, según en el grado de atipia nuclear y la cantidad de mitosis (Harrison et al., 2008). Los carcinomas serosos de bajo grado son poco frecuentes y generalmente asociados con los tumores limítrofes. Recientes investigaciones indican que el tubo ovárico es el lugar donde los carcinomas ováricos serosos se originan, especialmente en la fimbria tubárica que juega un papel importante en la carcinogénesis (Harrison et al., 2008).

Los carcinomas mucinosos se asocian a menudo con un cistoadenoma mucinoso y son con más frecuencia unilaterales, se los diagnostica en la etapa I. Los carcinomas endometrioides y de células claras se asocian con frecuencia con la endometriosis, que se considera generalmente un factor de riesgo de carcinoma de ovario. El 2\% de los pacientes presentan carcinoma de células claras, el 9\% carcinoma endometrioide y el $2 \%$ carcinoma seroso de bajo grado (Pearce et al., 2012).

\section{Estatificación del cáncer de ovario.-}

Para la estatificación del cáncer de ovario se utiliza el sistema TNM, que está basado en la extensión del tumor $(\mathrm{T})$, el grado de diseminación a los ganglios linfáticos $(\mathrm{N})$ y la presencia de metástasis distante $(\mathrm{M})$. Un número se añade a 
cada letra para indicar el tamaño o extensión del tumor y el grado de diseminación del cáncer (Singletary et al., 2002). Con respecto al tumor primario (T), se subdivide en: TX, el tumor primario no puede ser evaluado; T0, no hay evidencia de tumor primario; Tis, carcinoma in situ; T1, T2, T3, T4, tamaño y extensión del tumor primario. En cuanto a los ganglios linfáticos regionales $(\mathrm{N})$, se subdivide en: $\mathrm{NX}$, no es posible evaluar los ganglios linfáticos regionales; N0, no existe complicación de ganglios linfáticos; N1, N2, N3, complicación de ganglios linfáticos regionales. Número de ganglios linfáticos y grado de diseminación. Con relación a la metástasis distante $(\mathrm{M})$, se subdivide en: $\mathrm{MX}$, no es posible evaluar metástasis distante; $\mathrm{M} 0$, no existe metástasis distante; y M1, presencia de metástasis distante. Además, los estadios se subclasifican en: 0: carcinoma in situ; I, II y III, la enfermedad se torna más extensa y existe mayor tamaño tumoral; y IV, el cáncer se ha diseminado a otros órganos (National Cancer Institute, 2013; Woodward et al., 2003).

\section{Epidemiología.-}

La tasa de incidencia a nivel mundial es de 6,3 por cada 100.000 mujeres; la más alta en regiones más desarrolladas es de $(9,3)$ que en regiones menos desarrolladas $(5,0)$. El continente con la tasa más alta de incidencia es Europa $(10,1)$, seguido de América del Norte (8,7), Oceanía (7,6), América del Sur (6,2), América Central $(5,2)$, Asia $(5,1)$ y África (4,2) (Ferlay et al., 2010).

Con respecto a la mortalidad, la tasa mundial fue de 3,8 por cada 100.000 habitantes hasta el 2008. Los continentes con las tasas de mortalidad más altas son Europa y América del Norte $(5,4)$, Oceanía (4,7), América del Sur, América Central y África (3,4), y Asia (3,0) (Ferlay et al., 2010).

El cáncer de ovario es la sexta neoplasia más frecuente en mujeres en el Ecuador. La tasa de incidencia de cáncer de ovario ha ido en aumento con el pasar de los años; en el período 19861990 la incidencia era de 5,0 mientras que en el período 2001-2005 se incrementó a 7,4. En relación con la edad, la tasa de incidencia es 28,9 en mujeres mayores a 70 años, 7,5 en mujeres de 50 años y 1,6 en mujeres menores a 50 años (Cueva y Yépez, 2009). En el Ecuador, la tasa de mortalidad del total de los casos de cáncer de ovario es 2,9. Es decir, es menos frecuente pero más dañino debido a su diagnóstico tardío (Chobanian y Dietrich, 2008).

\section{Etiología.-}

Según la Organización Mundial de la Salud, un factor de riesgo es cualquier rasgo, característica o exposición de un individuo que aumente su probabilidad de sufrir una enfermedad o lesión (Organización Mundial de la Salud, 2009). Debido a la interacción entre los factores ambientales y la información genética, se sugiere que el cáncer es inducido por procesos que ocurren durante la transformación celular del tumor (Baba y Cătoi, 2007). Existen diversos factores asociados al desarrollo del cáncer de ovario:

\section{Edad.-}

El diagnóstico de este carcinoma se encuentra relacionado con mayor frecuencia en mujeres posmenopáusicas, a pesar de que la exposición a los factores de riesgo está presente con más fuerza en mujeres premenopáusicas (Hernández, 1999).

\section{Carga hormonal.-}

En el ovario las hormonas luteinizante (LH) y foliculoestimulante (FSH) actúan sobre las células de la granulosa y de la teca del ovario. Estas hormonas regulan la proliferación celular, apoptosis, adhesión celular y angiogénesis. Excesiva exposición de gonadotropinas aumenta la posibilidad de cáncer de ovario. En la menopausia se incrementa el riesgo de desarrollar este carcinoma debido al aumento de los niveles de gonadotropinas como consecuencia del cese de la función ovulatoria. Además, el uso de terapias con estrógenos, de productos hormonales farmacéuticos (anticonceptivos orales), y de la terapia remplazadora de hormonas (HRT), han sido asociados con el incremento en el desarrollo del cáncer de ovario y de mama (Pasalich et al., 2013; Moorman et al., 2008; Berstein, 2006).

\section{Obesidad.-}

Con relación a los efectos hormonales, el exceso de grasa incrementa la producción de estrógenos, 
propiciando el desarrollo del cáncer de ovario. Los lipocitos producen hormonas llamadas adipoquinas, que pueden estimular o inhibir el crecimiento celular (National Cancer Institute, 2013).

\section{Antecedentes familiares.-}

El patrón de herencia de este carcinoma es autosómico dominante. El presentar antecedentes familiares de cáncer de ovario aumenta el riesgo de padecer esta enfermedad del 1,5 al 4\%, mientras que el tener la presencia de esta enfermedad en dos familiares, aumenta su riesgo al 7\% (Shulman y Dungan, 2010).

\section{Agentes carcinogénicos.-}

Los carcinógenos son compuestos o sustancias que producen diferentes tipos de alteraciones en el organismo y generan el desarrollo del cáncer bajo una alta dosis y tiempo de exposición (Frank, 2007). En el ambiente laboral existen compuestos perjudiciales como son las fibras de asbesto, talco, metales pesados, pesticidas, arsénico, benceno, xileno, tolueno, aminas aromáticas, monoclorhidrato de vinilo, hidrocarburos, radiación ionizante y radiación ultravioleta. Igualmente, existen agentes patógenos asociados al desarrollo del cáncer como son los virus (EBV, VPH, VHB, VHC, VTLH-1 o HHV8), o bacterias como el Helicobacter pylori (Cabrera-Andrade et al., 2014; Paz-y-Miño et al., 2008). El humo del tabaco es uno de los carcinógenos más potentes debido a sus componentes mutágenos como el hidrocarburo aromático policíclico (PAH) y la nitrosamina (Paz-y-Miño et al., 2010; Frank, 2007; Irigaray et al., 2007). Otro ejemplo claro de agente carcinogénico son los rayos $\mathrm{X}$ aplicados en los procedimientos médicos o terapéuticos, donde el riesgo de adquirir cáncer incrementa conjuntamente con el tiempo de exposición de la radiación (Muñoz et al., 2008; Irigaray et al., 2007). Los agentes carcinogénicos mencionados anteriormente alteran el ADN y causan alteración en las funciones celulares; sin embargo, una predisposición hereditaria o genética ligada o no a estos agentes puede acelerar el proceso de desarrollo de cáncer (Frank, 2007).

\section{Perfil genético.-}

Dentro de la población femenina, hay ciertos grupos que pueden tener predisposición hereditaria y susceptibilidad a desarrollar cáncer. Existen factores de riesgo en los cuales se encuentran asociados diversos genes, y variaciones del ADN de estos genes predisponen a la mujer a desarrollar varios tipos de cáncer, entre ellos el de ovario. Algunos de estos son los siguientes:

\section{BRCA1}

Este gen se localiza en el cromosoma 17, en la banda 17q21. Las mutaciones se pueden generar en cualquiera de los 5500 pares de bases que conforma este gen, y su penetrancia es variable (Abe et al., 2014). El gen BRCA1 codifica una fosfoproteína nuclear que juega un papel importante en el mantenimiento de la estabilidad genómica, y también actúa como un gen supresor de tumores. Se expresa en distintos epitelios del organismo durante el desarrollo. Se ha observado que la activación del gen BRCA1 es inducida por estrógenos, y su inhibición causa un aumento de la proliferación celular del tejido mamario (Abe et al., 2014).

\section{BRCA2}

Este gen se localiza en el cromosoma 13, en la banda 13q12. Es considerado un gen supresor tumoral. Mutaciones en este gen están estrechamente relacionadas con el desarrollo del cáncer de mama y de ovario (Abe et al., 2014).

Los genes BRCA1 y BRCA2 constituyen casi el 90\% de los trastornos genéticos hereditarios responsables del cáncer de ovario. Las mutaciones germinales son responsables de cerca del $10 \%$ de los tumores malignos de este carcinoma. Se han descrito cerca de 460 diferentes mutaciones en el gen BRCA1 y cerca de 200 en el gen BRCA2, la mayoría de estas mutaciones $(80 \%)$ originan un codón de parada que lleva a la síntesis de una proteína truncada (Lengyel, 2010; Duarte et al., 2002).

\section{TP53}

Este gen se localiza en el cromosoma 17, en la banda 17p13. La transición al cáncer se caracteriza por el daño de los alelos y consecuente pérdida del gen funcional TP53. Mutaciones en este gen dan lugar al desarrollo de la mayoría de casos 
de cáncer de ovario, influyen en el ciclo celular de las células del ovario, en la inducción a la apoptosis y en la respuesta al estrés oxidativo, por lo cual la falla en la expresión de este gen tiene gran repercusión en el desarrollo del carcinoma de ovario (Chuaire-Noack, 2008).

Debido a que las mutaciones de TP53 son las más comunes en el ADN de los tumores de tipo II, es posible que el ADN tumoral se pueda detectar en los fluidos corporales. Los carcinomas de bajo y alto grado, presentan perfiles genómicos diferentes.

\section{RAD51}

Este gen se localiza en el cromosoma 15, en la banda 15q15. Participa en la síntesis de proteínas encargadas de reparar el ADN dañado a través del proceso de recombinación homóloga y de la interacción con otras proteínas reparadoras codificadas por los genes BRCA1 y BRCA2. Mutaciones en el gen RAD51 alterarán la síntesis de proteínas reparadoras y desencadenarán la acumulación de ADN dañado el cual podría desencadenarse en cáncer (National Cancer Institute, 2013).

\section{VDR}

Este gen se localiza en el cromosoma 12, en la banda 12q13. La vitamina D está implicada en una variedad de procesos biológicos como el metabolismo óseo, regulación de la respuesta inmune, proliferación y diferenciación celular. Existe una relación inversa entre los niveles de vitamina $\mathrm{D}$ en sangre y la incidencia de diversos tipos de cáncer (Grant, 2006). Se ha sugerido que una deficiencia de vitamina $\mathrm{D}$ puede ser un factor de riesgo para el desarrollo de cáncer de ovario, especialmente en mujeres con sobrepeso y obesidad (Mohapatra et al., 2013; Tworoger et al., 2007).

\section{Avances en el tratamiento.-}

El correcto tratamiento del cáncer de ovario dependerá del estadio tumoral, edad, capacidad de absorción de fármacos, histopatología, carga hormonal y perfil genético, mediante un tratamiento personalizado. A pesar de ello, existen diferentes métodos de tratamiento los cuales van desde medicina homeopática hasta inhibición de ARN mensajero mediante ribointerferencia.

\section{Medicina homeopática.-}

Bufalin es un componente inmunoreactivo obtenido de la piel y glándulas parótidas venenosas del sapo. Se ha demostrado que esta medicina tradicional oriental induce apoptosis en células cancerosas humanas y también inhibe el crecimiento de las células tumorales. Además, son considerados esteroides C-24 cardioactivos que poseen varias actividades biológicas; a pesar de esto puede ser utilizado por largos periodos sin efectos severos (Takai et al., 2012)

\section{Farmacología en el tratamiento de cáncer de ovario.-}

El tratamiento consiste en el uso de medicamentos cuya finalidad es asesinar a las células cancerosas y de igual manera reducir tumores (Tabla 1) (American Cancer Society, 2013). A menudo estos medicamentos son administrados por vía intravenosa, oral o administrada directamente a través del vientre. A menudo las drogas administradas pueden ser de 2 tipos durante un período de 3 a 4 semanas. Al mismo tiempo que este tratamiento asesina células cancerosas, células sanas se ven afectadas de igual forma, lo cual produce efectos secundarios, entre los más comunes podemos observar: pérdida de apetito, pérdida de cabello, vómito, erupciones en manos, pies y llagas en la boca (American Cancer Society, 2013). 
Tabla 1. Fármacos aprobados para el tratamiento del cáncer de ovario

Fármacos

Doxorrubicina

Carboplatina,

ciclofosfamina, cisplatino,

altrentamina

Gemcitabina

Paclitaxel

Capecitabina

Ifosfamida

Premetexed
Función

Retarda o detiene el crecimiento de las células cancerosas

Agente alquilante.

Detiene el crecimiento de las células cancerosas causando su muerte.

Previene que las células produzcan ADN y ARN y detienen el crecimiento celular.

Interfiere con los microtúbulos y produce la muerte celular.

Antimetabolito. Permite que el fármaco 5-fluoracil (5-FU) activado se dirija al tumor.

Agente alquilante. Reduce el crecimiento de las células cancerosas.

Antimetabolitos. Previene que las células utilicen folatos para formar ADN y ARN, con inhibición en el crecimiento de las células cancerígenas
Función de micro ARNs en el tratamiento de carcinoma ovárico.-

El cisplatino es el componente quimioterapéutico más eficaz contra el carcinoma ovárico, con un porcentaje inicial de respuesta del $40 \%$ al $80 \%$. Las terapias de combinación, en especial carboplatino con paclitaxel ofrecen una mejora significativa y este procedimiento se ha estandarizado para aquellas mujeres con cáncer de ovario epitelial avanzado. Desafortunadamente, muchas mujeres con tumores que responden inicialmente a una quimioterapia generan frecuentemente resistencia a los medicamentos. Dahiya y colaboradores han encontrado que 60 micro ARNs se encuentran inmersos en el proceso de inhibición de la resistencia a fármacos en el cáncer de ovario (Dahiya y Morin, 2010).

\section{Inhibidores multicinasas.-}

Sorafenib es un medicamento de administración oral que se encarga de inhibir las multicinasas, el cual se va a dirigir a la vía de mitógeno acti- vado de la proteína quinasa (MAPK) o RAF/ MEK/ERK. Este medicamento tiene la capacidad de inhibir a VEGFR 1, 2 y 3 y al receptor del factor de crecimiento derivado de plaquetas (PDGFR). Además, este medicamento ha sido aprobado por la FDA para el tratamiento del cáncer avanzado de células renales (Wilhelm et al., 2008).

\section{Avances quirúrgicos.-}

El rol de la cirugía en el cáncer de ovario ha sido una opción muy tomada en cuenta, pero no siempre fue la primera opción, al igual que la quimioterapia tiene sus efectos secundarios; entre uno de los más discutidos es la pérdida de fertilidad. Los estadios III y IV del cáncer de ovario son tratados quirúrgicamente, es de una histerectomía y una salpingooforectomía bilateral, incluida una omentectomía para evitar la diseminación (Al Rawahi, 2013). En el año 2012, el Centro del Cáncer MD Anderson llegó a la conclusión que la cirugía debe ser la 
primera opción con el fin de evitar la evolución progresiva del cáncer y posteriormente tratar al paciente con fármacos afines a su perfil genético (Vademecum, 2011).

\section{DISCUSIÓN}

Es innegable que las consecuencias ambientales ocasionadas por la industrialización en el último siglo han afectado drásticamente el ambiente y la salud humana, desarrollando diversos tipos de cáncer como el de ovario. El carcinoma ovárico es un tipo de neoplasia maligna que ocupa uno de los primeros lugares de incidencia en todo el mundo. Este cáncer se ha mantenido constante en países como Estados Unidos, y en otros ha ido en aumento su incidencia con el paso del tiempo, hasta alcanzar su pico a los 57 años de edad en mujeres blancas europeas y norteamericanas (Chobanian y Dietrich, 2008).

En esta revisión bibliográfica encontramos que existen varios factores epigenéticos que inducen al desarrollo del cáncer de ovario como son la edad, la dieta, la carga hormonal, la predisposición hereditaria, el grupo etario, la exposición a agentes carcinogénicos como los hidrocarburos aromáticos policíclicos, el consumo de tabaco (Modugno, 2002), el uso de talco, toallas sanitarias y compresas que contengan un compuesto similar al asbesto llamado carcinogen, sobre todo en mujeres en edad reproductiva (Mills et al., 2004; Harlow et al., 1992).

Los ovarios poseen varios tipos de células, las cuales pueden transformarse cada una en diferentes subtipos de cáncer, por lo cual se han podido identificar diferentes patrones histopatológicos que deben ser tratados de forma específica. Con los datos analizados histológicamente, este cáncer depende de las células afectadas y la proliferación que estás realicen (Smolle et al., 2013). Las distintas células afectadas favorecen al desarrollo de carcinomas que pueden ser unilaterales o bilaterales, tumores que solo se van a observar en los diferentes estadios de la enfermedad I, II, III, IV, que pueden concluir con una metástasis hacia zonas cercanas como el cérvix o el endometrio (Lee et al., 2003). Los carcinomas de tipo seroso abarcan el 50\% de cáncer de ovario, y estos son divididos en bajo grado y alto grado, basándose en la atipia nuclear y en la mitosis que presenten (Smolle et al., 2013; Pearce et al., 2012).

Con respecto al perfil genético, se ha podido evidenciar que la presencia de mutaciones clínicamente significativas en los genes TP53, VDR, mEH, BRCA1, BRCA2, RAD51 ocurren en 1 de cada 500 personas a nivel mundial, desencadenando el desarrollo del cáncer de ovario. La especificación y relación de los factores epigenéticos asociados a la enfermedad han sido de gran ayuda para aplicar principios de prevención en el tratamiento contra esta enfermedad. Por ejemplo, el estudio de observación de la ooforectomía indica un menor riesgo en el desarrollo del cáncer de personas portadoras de mutaciones en los genes BRCA1 y BRCA2. Además, el tratamiento preventivo con quimioprevención revela un número significativo de personas que no desarrollan el cáncer de ovario (Nagle et al., 2008).

Es interesante mencionar que los grandes avances científicos y tecnológicos han permitido una mejor interpretación acerca de la asociación de los factores ambientales en el desarrollo del carcinoma ovárico. Los avances en el tratamiento del cáncer de ovario presentados en este estudio tienen como finalidad disminuir la mortalidad y evitar la metástasis de las células afectadas hacia nuevas zonas. El uso de diferentes métodos, medicina homeópata, farmacología, avances genéticos y quirúrgicos, que se dan a conocer es válido en el tratamiento de la enfermedad. Tal es el caso de un estudio de cohorte realizado en la Universidad de Carolina del Norte, en donde se registró una asociación entre el consumo de fitoquímicos (fitoestrógenos o isotiocinatos) con los bajos niveles de cáncer de ovario (Chang et al., 2007). Las isoflavonas, una clase de fitoestrógenos que se encontraron en los alimentos a base de soya, tienen efectos antiestrogénicos y antiproliferativos (Cornwell et al., 2004), e inhiben el crecimiento y la proliferación de las células cancerígenas a través de la unión competitiva a los receptores de estrógeno o a través de la inhibición de la actividad de la aromatasa, la enzima responsable de la conversión de andrógenos a 
estrógenos (Chen et al., 2001; Kuiper et al., 1998; Pelissero et al., 1996). Los isotiocianatos y las isoflavonas que se encuentran en las verduras crucíferas colaboran en los procesos de desintoxicación de carcinógenos, protegen contra el daño oxidativo y ejercen efectos antiestrogénicos y controlan el desarrollo de tumores (Auborn et al., 2003; Lampe et al., 2002; Bradlow et al., 1999).

En conclusión, esta revisión bibliográfica acerca de la epidemiología, etiología, histopatología, tratamientos, factores ambientales y genéticos es de gran importancia para comprender las vías de desarrollo relacionadas con el carcinoma ovári$\mathrm{co}$, y por ende generar conciencia social para mejorar el estilo de vida, cuidar la salud y prevenir el desarrollo de esta enfermedad.

\section{REFERENCIAS BIBLIOGRÁFICAS}

Abe A, Sakamoto K, Taniguchi T, Nomura H, Yamamoto A, Okamoto S, Omatsu K, Kato K, Arai M, Takeshima N. 2014. The presentation of an asymptomatic ovarian carcinosarcoma caught by BRCA1 mutation surveillance program. International Cancer Conference Journal, DOI 10.1007/s13691014-0155-9.

Al Rawahi T, Lopes A, Bristow R, Brayant A, Elattar A, Chattopadhyay S, Galaal K. 2013. Surgical cytoreduction for recurrent epithelial ovarian cancer. John Wiley \& Sons Ltd. USA. 45 pp.

American Cancer Society. 2013. Ovarian Cancer. Página de Internet: http://www.cancer. org/cancer/ovariancancer/detailedguide/ index Consultada 10-diciembre-2013.

Auborn K, Fan S, Rosen E, Goodwin L, Chandraskaren A., William D., Chen D. y Carter T. 2003. Indole-3-carbinol is a negative regulator of estrogen. The Journal of Nutrition, 133(7 Suppl): 2470S-5S.

Baba A y Cătoi C. 2007. Comparative Oncology. The Publishing House of the Romanian Academy. Bucharest, Romania.
Bankhead C, Collins C, Stokes-Lampard H, Rose P, Wilson S, Clements A, Mant D, Kehoe S, Austoken J. 2008. Identifying symptoms of ovarian cancer: a qualitative and quantitative study. BJOG: An International Journal of Obstetrics and Gynaecology, 115(8): 1008-14.

Bast R, Hennessy B y Mills G. 2009. The biology of ovarian cancer: new opportunities for translation. Nature Reviews Cancer, 9(6): 415-28.

Bernstein L. 2006. The risk of breast, endometrial and ovarian cancer in users of hormonal preparations. Basic y Clinical Pharmacology y Toxicology, 98: 288-96.

Bradlow H, Sepkovic D, Telang N, Osborne M. 1999. Multifunctional aspects of the action of indole-3-carbinol as an antitumor agent. Annals of the New York Academy of Sciences, 889:204-13.

Cabrera-Andrade A, López-Cortés A, Muñoz M. J, Jaramillo-Koupermann G, Rodríguez O, Leone P. E, Paz-y-Miño C. 2014. Association of genetic variants of membrane receptors related to recognition and induction of immune response with Helicobacter pylori infection in Ecuadorian individuals. International Journal of Immunogenetics, DOI: 10.1111/iji.12118.

Chang E, Lee V, Canchola A, Clarke C, Purdie D, Reynolds P, Anton-Culver H, Bernstein L, Deapen D, Peel D, Pinder R, Ross R, Stram D, West D, Wright W, Ziogas A, HornRoss P. 2007. Diet and risk of ovarian cancer in the California Teachers Study cohort. American Journal of Epidemiology, 165(7):802-13.

Chen H, Hardy T y Tollefsbol T. 2011. Epigenomics of ovarian cancer and its chemoprevention. Frontiers in Genetics, 2:67.

Chen X y Anderson J. 2001. Isoflavones inhibit proliferation of ovarian cancer cells in 
vitro via an estrogen receptor-dependent pathway. Nutrition and cancer, 41:165-71.

Chobanian Ny Dietrich Ch. 2008. Cáncer de ovario. Surgical Clinics of North America, 45:149-166.

Chuaire-Noack L, Sánchez-Corredor M, Ramírez-Clavijo S. 2008. P53 and its role in the ovarían surface epithelium. A review. Investigación Clínica, 49(4): 561-93.

Clavel J. 2007. Progress in the epidemiological understanding of gene-environment interactions in major diseases: cancer. Comptes Rendus Biologies, 330(4): 306-17.

Cornwell T, Cohick W, Raskin I. 2004. Dietary phytoestrogens and health. Phytochemistry, 65: 995-1016.

Cueva P y Yépez J. 2009. Epidemiología del cáncer en Quito 2003-2005. Registro Nacional de Tumores (RNT). SOLCA. Quito, Ecuador. 200 pp.

Dahiya N y Morin P. 2010. MicroRNAs in ovarian carcinomas. Endocrine-Related Cancer, 17(1): 77-89.

Duarte F, Camesselle-Teijeiro J, Soares R, Seixas C, Cortizo-Torres M. 2002. Análisis de mutaciones en los genes BRCA1 y BRCA2 en pacientes con cáncer de mama y ovario del norte de Portugal y Galicia. Revista Clínica Española, 202(5): 259-63.

Ferlay J, Shin H, Bray F, Forman D, Mathers C, Parkin D. 2010. Estimates of worldwide burden of cancer in 2010: GLOBOCAN 2008. International Journal of Cancer, 127(12): 2893-917.

Frank S A. 2007. Dynamics of cancer incidence, inheritance, and evolution. Princeton University Press. Princeton and Oxford. United Kingdom. 273 pp.

Grant W. 2006. Lower vitamin-D production from solar ultraviolet-B irradiance may explain some differences in cancer survival rates. Journal of the National Medical Association, 3(6): 357-64.

Harlow B, Cramer D, Bell D, Welch W. 1992. Perineal exposure to talc and ovarian cancer risk. Obstetrics and Gynecology, 80(1): 19-26.

Harrison M., Jameson C., Gore M. 2008. Mucinous ovarian cancer. International Journal of Gynecological Cancer, 18(2): 209-14.

Hernández M y Ríos M. 1999. Oncogenes y Cáncer. Revista Cubana de Oncología, 15(2): 131-9.

Irigaray P, Newby J, Clapp R, Hardell L, Howard V, Montagnier L, Epstein S y Belpomme D. 2007. Lifestyle-related factors and environmental agents causing cancer: an overview. Biomedicine y Pharmacotherapy, 61(10): 640-58.

Karst A y Drapkin R. 2010. Ovarian cancer pathogenesis: a model in evolution. Journal of Oncology, 1-13.

Kuiper G, Lemmen J, Carlsson B, Corton J, Safe S, van der Saag P, van der Burg y Gustafsson J. 1998. Interaction of estrogenic chemicals and phytoestrogens with estrogen receptor beta. Endocrinology, 139(10): 4252-63.

Kurman R y Shih I. 2008. Pathogenesis of ovarian cancer: lessons from morphology and molecular biology and their clinical implications. International Journal of Gynecological Pathology, 27(2): 151-60.

Kwon M y Shin Y. 2011. Epigenetic regulation of cancer-associated genes in ovarian cancer. International Journal of Molecular Sciences, 12(2): 983-1008.

Lagos E y Soto T. 2007. Epigenética y cáncer. Revista Médica de Costa Rica y Centroamerica, 580: 177-182. 
Lampe J y Peterson S. 2002. Brassica, biotransformation and cancer risk: genetic polymorphisms alter the preventive effects of cruciferous vegetables. The Journal of Nutrition, 132(10): 2991-4.

Lee K y Young R. 2003. The distinction between primary and metastatic mucinous carcinomas of the ovary: gross and histologic findings in 50 cases. The American Journal of Surgical Pathology. 27(3): 281-92.

Lengyel E. 2010. Ovarian cancer development and metastasis. The American Journal of Pathology, 177(3): 1053-64.

Mills P, Riordan D, Cress R y Young H. 2004. Perineal talc exposure and epithelial ovarian cancer risk in the Central Valley of California. International Journal of Cancer, 112(3): 458-64.

Modugno F, Ness R y Cottreau C. 2002. Cigarette smoking and the risk of mucinous and nonmucinous epithelial ovarian cancer. Epidemiology, 13:467-71.

Mohapatra S, Saxena A, Gandhi G, Koner B y Ray P. 2013. Vitamin D and VDR gene polymorphism (FokI) in epithelial ovarian cancer in Indian population. Journal of Ovarian Research, 6(1): 37.

Moore K. 2010. Clinically Oriented Anatomy. Sixth Edition. Lippincott Williams and Wilkins. USA.

Moorman P, Claingaert B, Palmieri R, Iversen E, Bentley R, Halabi S, Berchuck A y Schildkraut J. 2008. Hormonal risk factors for ovarian cancer in premenopausal and postmenopausal women. American Journal of Epidemiology, 167(9): 1059-69.

Muñoz MJ, López-Cortés A, Sarmiento I, Herrera C, Sánchez ME y Paz-y-Miño C. 2008. Biomonitoreo genetico de individuos expuestos a radiación ionizante y su relación con el desarrollo de cancer. Oncología, 18(1-4):75-82.
Nagle C, Olsen C, Webb P, Jordan S, Whiteman D, Green A, Australian Cancer Study Group y Australian Ovarian Cancer Study Group. 2008. Endometrioid and clear cell ovarian cancers: a comparative analysis of risk factors. European Journal of Cancer, 44(16): 2477-84.

National Cancer Institute. 2013. Cáncer epitelial de ovarios: Tratamiento. National Institute of Health. Página de Internet: http:/ / www. cancer.gov/espanol/pdq/tratamiento/ epitelial-de-ovarios / HealthProfessional/ page5 Consultada 5-noviembre-2013.

Nicolopoulou-Stamati P, Hens L, Howard V y van Larebeke N. 2004. Cancer as an Environmental Disease. Environmental Science and Technology Library. Kluwer Academic Publishers. Holanda.

Organización Mundial de la Salud (OMS). 2009. Cancer: Fact sheet 292. Página de Internet: http:/ / www.who.int/mediacentre/factsheets/fs297/en/ Consultada 30mayo-2011.

Pasalich M, Su D, Binns C y Lee A. 2013. Reproductive factors for ovarian cancer in southern Chinese women. Journal of Gynecologic Oncology, 24(2): 135-40.

Patel A, Galaal K, Burnley C, Faulkner K, Martin-Hirsch P, Bland M, Leeson S, Beer H, Paranjothy S, Sasieni P y Naik R. 2012. Cervical cancer incidence in young women: a historical and geographical controlled UK regional population study. British Journal of Cancer, 106(11): 1753-9.

Paz-y-Miño C, López-Cortés A, Muñoz MJ, Cabrera A, Castro Bernardo, Sánchez M E. 2010. Incidence of the L858R and G719S mutations of the epidermal growth factor receptor oncogene in an Ecuadorian population with lung cancer. Cancer Genetics and Cytogenetics, 196: 201-3. 
Paz-y-Miño C, López-Cortés A, Arévalo M, Sánchez ME. 2008. Monitoring of DNA damage in exposed individuals to petroleum hydrocarbons in Ecuador. Annals of the New York Academy of Sciences, 1140: 121-8.

Pearce C, Templeman C, Rossing M, Lee A, Near A, Webb P, Nagle C, Doherty J, Cushing-Haugen K, Wicklund K., et al. 2012. Association between endometriosis and risk of histological subtypes of ovarian cancer: a pooled analysis of case-control studies. The Lancet Oncology, 13(4): 385-94.

Pelissero C, Lenczowski MJ, Chinzi D, Davail-Cuisset B, Sumpter J, Fostier A. 1996. Effects of flavonoids on aromatase activity, an in vitro study. The Journal of Steroid Biochemistry and Molecular Biology, 57(3-4): 215-23.

Sánchez P y Gómez A. 2005. Cáncer de Ovario. Medicine, 1667-74.

Shulman L y Dungan J. 2010. Cancer genetics: risks and mechanisms of cancer in women with inherited susceptibility to epithelial ovarian cancer. Cancer Treatment and Research, 156: 69-85.

Singletary S, Allred C, Ashley P, Bassett L, Berry D, Borgen P, Clark G, Edge S, Hayes D, Hughes L, Hutter R, Morrow M, Page D, Recht A, Theriault R, Thor A, Weaver D, Wlieand H y Greene F. 2002. Revision of the American Joint Committee on Cancer staging system for breast cancer. Journal of Clinical Oncology, 20(17): 3628-36.

Smolle E, Taucher V, Pichler M, Petru E, Lax S, Haybaeck J. 2013. Targeting signaling pathways in epithelial ovarian cancer. International Journal of Molecular Sciences, 14(5): 9536-55.
Takai N, Kira N, Ishii T, Yoshida T, Nishida Y, Nasu K y Narahara H. 2012. Bufalin, a traditional oriental medicine, induces apoptosis in human cancer cells. Asian Pacific Journal of Cancer Prevention, 13(1): 399-402.

Tworoger S, Lee I, Buring J, Rosner B, Hollis B y Hankinson S. 2007. Plasma 25-hydroxyvitamin D and 1,25-dihydroxyvitamin D and risk of incident ovarian cancer. Cancer Epidemiological, Biomarkers \& Prevention, 16(4): 783-8.

Vademecum. 2011. Vademecum International 2011. Editorial Medicom. Barcelona, España.

Wilhelm S, Adnane L, Newell Ph, Villanueva A, Llovet J, Lynch M. 2008. Preclinical overview of sorafenib, a multikinase inhibitor that targets both Raf and VEGF and PDGF receptor tyrosine kinase signaling. Molecular Cancer Therapeutics, 7: 3129-40.

Woodward W, Strom E, Tucker S, McNeese M, Perkins G, Schechter N, Singletary S, Theriault R, Hortobagyi G, Hunt K, Buchholz T. 2003. Changes in the 2003 American Joint Committee on Cancer staging for breast cancer dramatically affect stage-specific survival. Journal of Clinical Oncology, 21(17): 3244-8.

Wrzeszczynski K, Varadan V, Byrnes J, Lum E, Kamalakaran S, Levine A, Dimitrova N, Zhang M y Lucito R. 2011. Identification of tumor suppressors and oncogenes from genomic and epigenetic features in ovarian cancer. PLOS One, 6(12): e28503. 